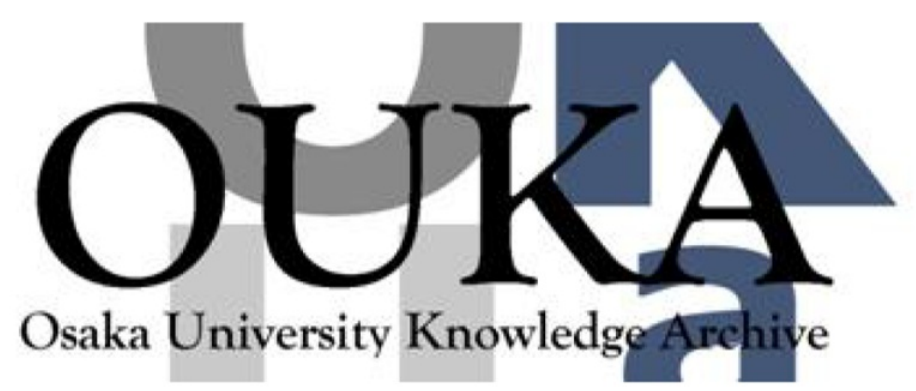

\begin{tabular}{|c|l|}
\hline Title & Structure of laboratory ball lightning \\
\hline Author(s) & $\begin{array}{l}\text { Ito, Tsuyohito; Tamura, Tomoya; Cappelli, Mark } \\
\text { A. et al. }\end{array}$ \\
\hline Citation & $\begin{array}{l}\text { Physical Review E - Statistical, Nonlinear, and } \\
\text { Soft Matter Physics. 80(6) p.067401 }\end{array}$ \\
\hline Issue Date & $2009-12-07$ \\
\hline oaire:version & VoR \\
\hline URL & https://hdl. handle. net/11094/78475 \\
\hline rights & $\begin{array}{l}\text { Copyright (2009) by the American Physical } \\
\text { Society" }\end{array}$ \\
\hline Note & \\
\hline
\end{tabular}

Osaka University Knowledge Archive : OUKA

https://ir. Library. osaka-u. ac. jp/

Osaka University 


\title{
Structure of laboratory ball lightning
}

\author{
Tsuyohito Ito, ${ }^{1, *}$ Tomoya Tamura, ${ }^{2}$ Mark A. Cappelli, ${ }^{3, \dagger}$ and Satoshi Hamaguchi ${ }^{2, \ddagger}$ \\ ${ }^{1}$ Frontier Research Base for Global Young Researchers, Frontier Research Center, Graduate School of Engineering, Osaka University, \\ 2-1 Yamadaoka, Suita, Osaka 565-0871, Japan \\ ${ }^{2}$ Center for Atomic and Molecular Technologies, Graduate School of Engineering, Osaka University, 2-1 Yamadaoka, Suita, \\ Osaka 565-0871, Japan \\ ${ }^{3}$ Mechanical Engineering Department, Stanford University, Stanford, California 94305-3032, USA \\ (Received 22 August 2009; revised manuscript received 27 October 2009; published 7 December 2009)
}

\begin{abstract}
Trajectories of self-sustained laboratory ball lightning, generated by arc discharges with silicon, are investigated for understanding the possibility of buoyant flight. Extremely low apparent densities are found, nearly approaching that of standard air. The freely buoyant balls are observed to survive for about $0.1 \mathrm{~s}$, with significantly buoyant balls surviving for several seconds. These ball lightning objects are found to have a density and size that can easily allow them to be carried by a gentle breeze of a few meters per second. The results are interpreted by a model that is an extension of that first proposed by Abrahamson and Dinniss [J. Abrahamson and J. Dinniss, Nature (London) 403, 519 (2000)]. The buoyant behavior of ball lightning seen in our experiments is believed to arise as a result of the formation of a nanoparticle oxide network growing from a molten silicon core.
\end{abstract}

DOI: 10.1103/PhysRevE.80.067401

PACS number(s): 52.80.Mg, 92.60.Pw

Sightings of ball lightning-bright light-emitting buoyant objects that survive in air for up to several seconds-have been reported for centuries. According to some surveys [1-3], ball lightning is almost always sighted together with natural lightning events. In fact, $95 \%$ of all ball lightning events are reported to have occurred during thunderstorms, and nearly $80 \%$ of ball lightning was seen to occur right after flashes of lightning, most of which were to the ground. It has been widely believed that ball lightning is initiated by natural lightning strikes.

In laboratory experiments, two types of luminous objects that behave in ways similar to those reported for ball lightning have been produced. One is those sustained by external energy sources (such as microwaves) [4-7] and the other is those sustained by the release of internal potential energy (such as chemical oxidation reactions) [8-10]. In this Brief Report we discuss the latter.

Abrahamson and Dinniss [8] suggested that ball lightning may be caused by a strike of natural lightning against the ground, which vaporizes (or liquefies) silicon that is naturally present in the soil. Vaporized (or liquefied) silicon then oxidizes and generates more heat due to self-sustaining exothermic oxidation reactions [11]. This combustion process causes bright light emission. These researchers also suggested that silicon vapor and reaction products (i.e., oxides) are quenched when they are cooled and form networks of silicon/silicon oxide nanoparticles that are easily convected by air currents.

More recently, Paiva et al. experimentally produced longlived silicon-based luminous balls appearing similar to ball lightning in nature [9]. In the studies of Ref. [9], an atmo-

\footnotetext{
*Corresponding author. tsuyohito@wakate.frc.eng.osaka-u.ac.jp

†hamaguch@ppl.eng.osaka-u.ac.jp
}

spheric pressure arc discharge between a tungsten or graphite electrode and a silicon wafer is used to violently melt and vaporize silicon. Stephan and Massey [10] produced siliconbased luminous balls using a similar arc discharge on silicon wafers and found that the spectrum of the bright white emission roughly matches that of a blackbody at a temperature in the range of 2800-3400 K. The nanoparticle structure as originally proposed by Abrahamson and Dinniss [8] would not survive at these high temperatures. Clearly, the structure would have to be modified to account for the ability of reach temperatures exceeding the melting point of silicon. Our experimental measurements described below can be explained if the ball lightning consists of a structure of a nanoparticle network growing from a molten silicon core.

Our approach in producing the ball lightning that we describe here is similar to these prior experiments $[9,10]$. We note, however, that these prior studies lack detailed experimental evidence confirming buoyant flight or the structure that can explain such a flight, particularly the ball density and size.

Understanding the possibility of buoyant flight requires knowledge of the structure of the luminous objects, including their apparent density and diameter. Such information can be extracted by an analysis of their trajectories. In our experiments, as schematically illustrated in Fig. 1, luminous objects are generated by discharges with silicon using a method similar to that of Ref. [9], with arc discharges in silicon between two graphite electrodes. Unlike the previously reported method $[9,10]$, we used small grains of $p$-type silicon of about 1-2 $\mathrm{mm}$ in size as seed material, which seems to make it easier to produce the luminous ball lightninglike objects.

To generate these luminous balls, we first short circuit the arc power supply operating in a current-regulated mode across the silicon grains to heat up the grains with a current of approximately $95 \mathrm{~A}$ (voltage of approximately $10 \mathrm{~V}$ ) for about $5 \mathrm{~s}$. We then slowly raise the upper graphite electrode ( $5 \mathrm{~mm}$ in diameter) to generate an arc discharge for approxi- 


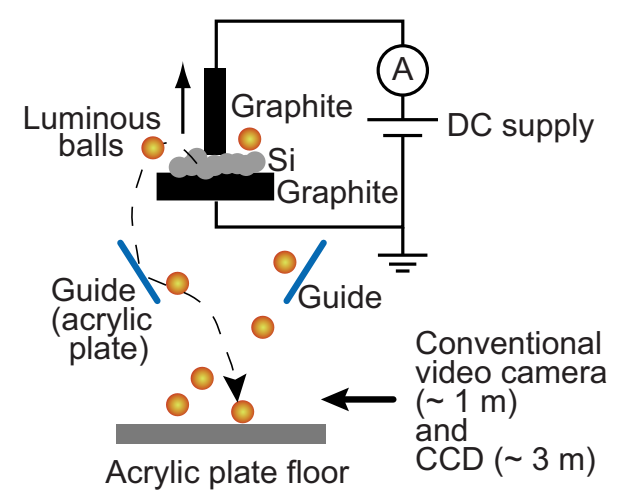

FIG. 1. (Color online) Schematic of the experimental setup. The voltage is typically about $10 \mathrm{~V}$, initially driving $\sim 95$ A through the silicon powder between the graphite electrodes.

mately $0.5 \mathrm{~s}$, which melts (and may even vaporize) the silicon fragments. The discharge generates molten silicon fragments that are illuminated for several seconds. Luminous balls (i.e., glowing silicon grains) initially drift by the gravitational force into the observation region just above an acrylic plate located $80 \mathrm{~cm}$ below the arc discharge generator. Their sizes and trajectories are recorded using conventional video and gated charge-coupled device (CCD) cameras.

Some cropped images of the buoyant luminous balls, as recorded by the conventional video camera, are provided in Fig. 2, in which a luminous ball accelerating upward is clearly seen after hovering at an almost constant height. Similar phenomena of upward acceleration were observed for many luminous balls. The trajectory, extracted velocity, and acceleration during "bounces" (in this case nine bounces by counting the first point as one bounce) of a single luminous ball within a $4 \mathrm{~cm}$ range above the acrylic plate are shown in Fig. 3(A). Examples of cropped images, as recorded by the CCD camera, are provided in Fig. 3(B), with the vertical dotted lines serving to define, based on the boundary of the luminous region, the apparent object size. Some examples of movies, recorded by the conventional

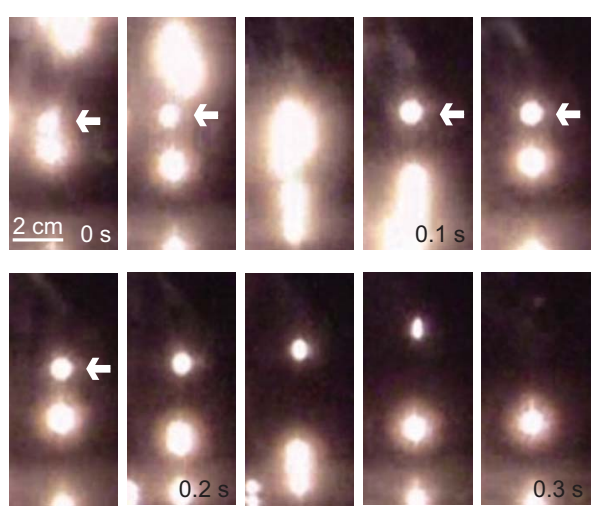

FIG. 2. (Color online) Video frames (from left to right, top to bottom) of luminous silicon balls taken by a conventional video camera at 30 frames/s. The small ball, indicated by arrows in several frames, is hovering at almost constant height in the first and fifth frames and accelerating upward in the fifth to ninth frames.
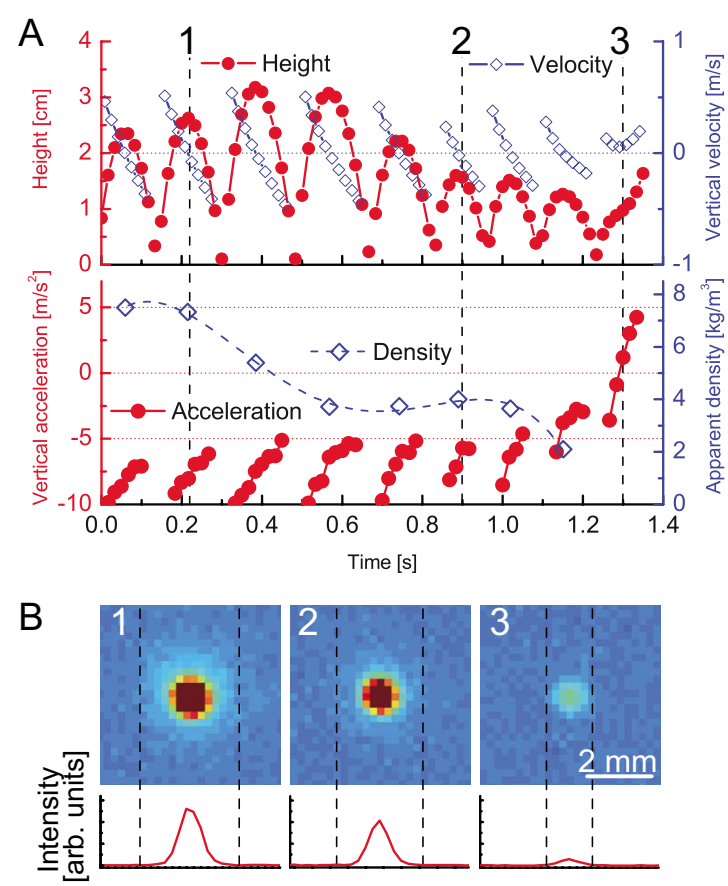

FIG. 3. (Color online) (A) The position, vertical velocity, vertical acceleration, and the apparent density as functions of time obtained from the trajectory of a silicon-based luminous ball. The CCD images of the silicon-based luminous ball at the time instances indicated by 1,2 , and 3 are given in (B), where the set of black broken lines indicate the "apparent diameter" of the luminous ball at each time instance.

video camera, are provided as supplemental movies 1 and 2 [12].

The most compelling data pointing to the existence of a strong buoyant force appear in Fig. 3(A), especially just before extinction. At first glance, the trajectories resemble that of a near-elastic solid bouncing from a floor; however, we see that successive bounces sometimes increase the peak height (first four bounces) and, ultimately, we see a strong concavity in the velocity, with an upward trajectory beyond the expected maximum. The main reason for the increase in the peak height in the ball's trajectories is not attributed to buoyancy, but rather to the removal of the oxidized nanoparticle network (evidence of which will be described later) during collisions with the floor. This exposes a molten silicon core near the bottom and results in nonisotropic oxidation reactions and a sudden vertical acceleration, as proposed in Ref. [10]. It is noteworthy that in studies reported in Ref. [10], the authors suggested that successive bouncing requires a refractory material (concrete), in contrast to our findings, in which we use an acrylic plate of relatively low melting temperature. We generally do not see any evidence of melting on the acrylic plate after successive bouncing, although sometimes the molten silicon drops are found to have solidified and attached to the plate and, in these rare cases, we do see clear evidence of melted acrylic (one can see most of the luminous balls bouncing successively in the supplemental movies 1 and 2 [12]). The particle acceleration is seen to be nearly always less than that of gravity, achieving a net acceleration opposite to that of gravity following its last bounce 

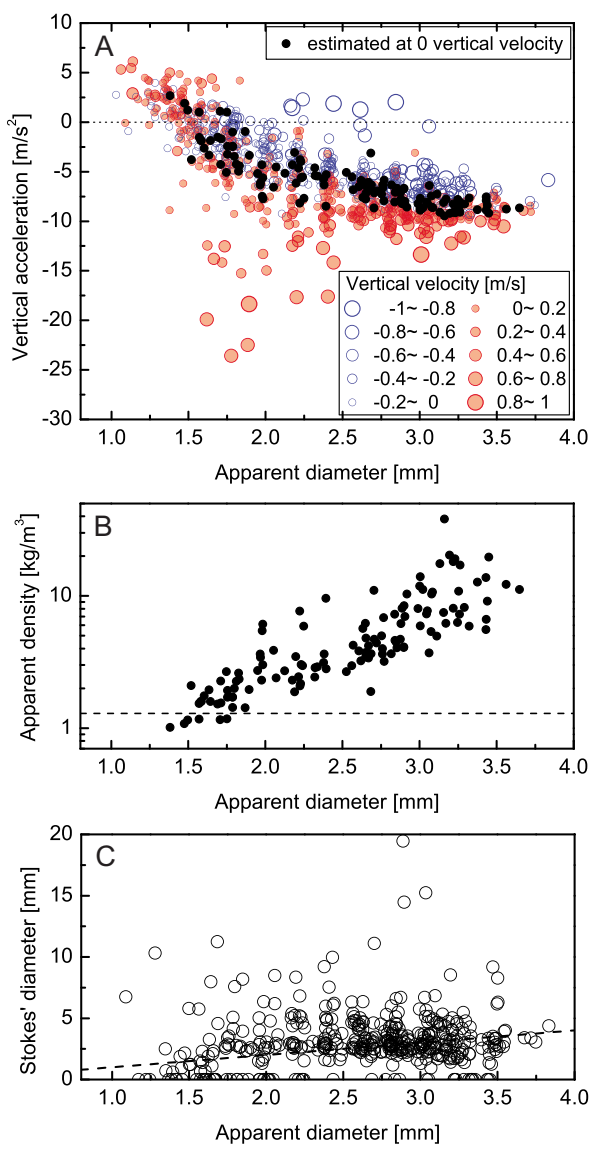

FIG. 4. (Color online) Values as functions of the apparent diameters recorded by the CCD camera: (A) vertical acceleration, (B) apparent density, and (C) diameter estimated from Stokes force. In (A) and (B), black filled circles represent values measured at zero velocity, and the color and size of the open circles indicate direction and magnitude of velocity. In (B) the broken line represents the density of air. In $(\mathrm{C})$, complex numbers caused by negative resistances are indicated as zero.

before extinction. These results confirm the existence of a substantial buoyant force that counteracts gravity and a Stokes drag force, proportional to the particle velocity that opposes motion. The apparent density of the particles can be extracted from when the particles have zero velocity, at which point only the buoyant force opposes gravity to establish the particle's acceleration. This density is also illustrated in Fig. 3(A), and we see that the upward motion following the final bounce occurs when the particles' apparent density approaches that of standard air $\left(1.29 \mathrm{~kg} / \mathrm{m}^{3}\right)$.

The acceleration is strongly correlated with the apparent diameter, illustrated in Fig. 4(A), as determined from the trajectories of 40 different luminous balls. The correlation depends weakly on velocity, depicted by symbol size and color. We see very clearly that the acceleration strongly depends on the diameter and we find that, when the diameter is less than $1.5 \mathrm{~mm}$, the particles exhibit positive acceleration, i.e., a net upward force. The upward acceleration is concomitant with a transition to a density that approaches that of air, as seen in Fig. 4(B). In general, we see a strong correlation between particle size and density, i.e., diminishing size results in diminishing density.
The diameters of the particles can be independently estimated from the extracted force due to drag estimated from regions in the particle flight where there is nonzero velocity. A comparison between this diameter, referred to as the Stokes diameter, and that measured using gated imaging is made in Fig. 4(C). Exact agreement would have these data following the dashed line depicted. No correction is made in our analyses of our experiments for the possibility of an upward draft, as such a draft was not seen in the laboratory during these studies. While we find that the period over which the particles have densities lighter than air is only about $0.1 \mathrm{~s}$ (see Fig. 2), it is noteworthy that over a larger part of the particles' lifetime, the particle's diameters and associated densities fall within a range that can easily allow them to be carried upward by a gentle breeze (due to the Stokes drag force) of a few meters per second. It is possible that, in natural phenomena, the lifetime of a buoyant luminous particle may be greatly extended beyond that time for which it has a density less than that of the surrounding air.

How is it that a silicon-based luminous particle can have such a low density, nearly approaching that of standard air? We conjecture that the structure of the particles comprises that of a molten silicon core, likely to be responsible for the bright white emission, surrounded by a gaseous halo trapped within a porous nanoparticle network - a structure similar to that proposed in Ref. [8]. Preliminary measurements made in our laboratory of the broadband thermal emission using a spectrometer in a wavelength range of 350-785 nm support the temperatures reported by Stephan and Massey [10]. The growth of the surrounding nanoparticle network can be supported by an oxide-assisted vapor-liquid-solid process [13], with $\mathrm{Si}$ and $\mathrm{SiO}$ vapor evolving from the molten particle surface as a result of partial oxidation. The oxidation and heating of the silicon core provides the precursors needed for the growth of the nanoparticle network, which can trap the hot gases, producing a lighter-than-air particle. For example, a particle consisting of a $100 \mu \mathrm{m}$ diameter core surrounded by a $1.5 \mathrm{~mm}$ diameter $90 \%$ porous nanowire network trapping heated vapor of $1000 \mathrm{~K}$ can readily achieve neutral buoyancy. The trapping of the hot vapor products is a result of the limited Knudsen diffusion through the porous network, and such a network can trap gases for time scales comparable to the times recorded for the buoyant flight of the lowest density particles followed in these experiments.

The lifetime of a typical luminous ball, in the experiments described above, is up to approximately $2 \mathrm{~s}$. Figure 5 depicts the lifetime of the luminous balls that bounce successively off of the acrylic plate, determined as it depends on the apparent diameter. We suspect that the balls survive longer if they spend more time in flight and avoid experiencing several bounces, as we believe that the relatively strong collisions with the floor results in the removal of the nanoparticle network that surrounds the core. This erosion is suspected to result in an increased exposure of the core to the surrounding air, and hence faster surface oxidation reactions. We note that luminous balls with lifetimes as high as $8 \mathrm{~s}$ have been reported in Ref. [9], and we confirm in our experiments that luminous balls of lifetimes of about $6 \mathrm{~s}$ can also be produced (supplemental movie 3 [12]) but without a large vertical component of velocity (without experiencing a strong impact with the floor). 


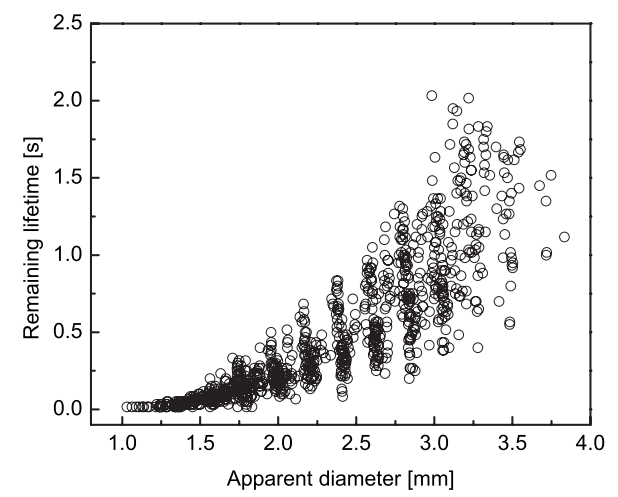

FIG. 5. Remaining luminous ball lifetime as a function of the apparent diameter (examples of the luminous bouncing silicon ball lightning can be seen in Fig. 3 and the supplemental movies 1 and $2[12])$.

To summarize, we have demonstrated that the siliconbased luminous balls with long lifetimes seem to have low apparent density, approaching that of standard air. The freely buoyant objects have been observed to survive for about 0.1 s, with significantly buoyant objects surviving for several seconds. These ball lightning objects are found to have a density and size that can allow them to be carried by a gentle breeze of a few meters per second, which may account for their convected flight in natural observances. We speculate that the structure may include a nanoparticle network growing from a molten silicon core. Although we have demonstrated persistent buoyancy following the generation of the luminous silicon balls, a direct connection to the phenomenon seen in nature requires a demonstration of similar behavior with arc discharge interactions with simulated ground soil (containing considerable silicon oxides). Experiments of this sort, with mixtures including oxides and also carbon are ongoing in our laboratories.

This research was performed at the Frontier Research Base for Global Young Researchers, Osaka University, with funding through the program Promotion of Environmental Improvement to Enhance Young Researchers' Independence, the special coordination funds for promoting science and technology, Japan Ministry of Education, Culture, Sports, Science and Technology (MEXT). This research has been funded by the Japan Society of Promotion of Science (JSPS)/ MEXT as well as the Murata Science Foundation. We would like to thank students who participated in the "Sosei" program in the Departments of Materials and Manufacturing Science, Graduate School of Engineering, Osaka University, for their technical support.
[1] M. Stenhoff, Ball Lightning: An Unsolved Problem in Atmospheric Physics (Kluwer Academic/Plenum Publishers, New York, 1999).

[2] J. R. McNally, Oak Ridge National Laboratory Report No. ORNL-3938, 1966 (unpublished).

[3] W. D. Rayle, National Aeronautics and Space Administration (NASA) Technical Note No. NASA-TN-D-3188 (NASA, Washington, D.C., 1966).

[4] P. L. Kapitza, Zh. Eksp. Teor. Fiz. 57, 1801 (1969) [Sov. Phys. JETP 30, 973 (1970)].

[5] Y. H. Ohtsuki and H. Ofuruton, Nature (London) 350, 139 (1991).

[6] V. Dikhtyar and E. Jerby, Phys. Rev. Lett. 96, 045002 (2006).

[7] J. B. A. Mitchell, J. L. LeGarrec, M. Sztucki, T. Narayanan, V. Dikhtyar, and E. Jerby, Phys. Rev. Lett. 100, 065001 (2008).
[8] J. Abrahamson and J. Dinniss, Nature (London) 403, 519 (2000).

[9] G. S. Paiva, A. C. Pavão, E. Alpes de Vasconcelos, O. Mendes, Jr., and E. Felisberto da Silva, Phys. Rev. Lett. 98, 048501 (2007).

[10] K. D. Stephan and N. Massey, J. Atmos. Sol.-Terr. Phys. 70, 1589 (2008).

[11] M. W. Chase, NIST-JANAF Thermochemical Tables I: Al-Co, 4th ed. (American Chemical Society and American Institute of Physics, New York, 1998).

[12] See EPAPS Document No. E-PLEEE8-80-168911 for supplemental movies showing the luminous balls. For more information on EPAPS, see http://www.aip.org/pubservs/epaps.html.

[13] Y. Yao, F. Lib, and S.-T. Lee, Chem. Phys. Lett. 406, 381 (2005). 